\title{
Virtual Singular Scattering of Electromagnetic Waves in Transformation Media Concept
}

\author{
Yurii Barabanenkov", Mikhail Barabanenkov", Sergey Nikitov ${ }^{1}$ \\ ${ }^{1}$ V.A Kotelnikov Institute of Radioengineering and Electronics, Russian Academy of Sciences, Moscow, Russia \\ ${ }^{2}$ Institute of Microelectronics Technology, Russian Academy of Sciences, Chernogolovka, Moscow, Russia \\ *corresponding author, E-mail: barab@iptm. ru
}

\begin{abstract}
A phenomenon of virtual singular scattering with multiplicative effect has been revealed while studying electromagnetic wave multiple scattering on a dielectric scatterer embedded into a flat left handed material slab (Veselago's lens) by the usual scattering operator ( $\mathrm{T}$ matrix) technique. If both a scatterer and an observation point (receiver) approach the so-called near field zone of a source of electromagnetic waves, the scattering process becomes a singular one which is mathematically attributed to the spatial singularity of the free space Green function at the origin. Virtual singular scattering means that a scatterer is only virtually situated in the near field zone of a source, being, in fact, positioned in the far field zone. Such a situation is realized if the inner focus of Veselago's lens approaches the position of a scatterer. Veselago's lens transfers the singularity of the free space Green function by implementing the coordinate transformation which results in the formation of virtual sources inside and behind the slab and virtual scatterers (as a source of secondary waves) on both slab sides. Considering a line-like dielectric scatterer, we demonstrate that the scattering efficiency is proportional to the product of singular quasistatic parts of two empty space Green functions, which means a multiplicative quasistatic singularity of the Green function for a slab of inhomogeneous Veselago medium. The appearance of virtual scatterers outside the slab involves both the inside and outside lens focuses. This circumstance physically distinguishes the multiplicative effect from the well known superlensing effect utilizing only the outer lens focus. We show that small inhomogeneity contributes significantly into the scattered field in the regime similar to the well known Mie resonance scattering.
\end{abstract}

\section{Introduction}

Newly proposed transformation optics concept [1,2] assumes that the space for electromagnetic (EM) field can be bent in an almost arbitrary way (in the absence of gravity) by filling EM space with spatial transformation media. Transformation media would implement the coordinate transformation, squeezing the originally flat electromagnetic space and guiding the light along curved trajectories [3].
We define the abovementioned transformation media concept as "newly" because this idea is not new. The origin of the concept can be found in the general relativity stating that given a curved space-time with a metric tensor, Maxwell's equations may be written as if they were valid in a flat-time in which there is an optical medium with a constitutive equation [4]. Answering the question with whom the idea that gravitation is equivalent to an optical medium originated, F. de Felice [4] wrote that perhaps A.Einstein was the first. Later on in 1923 Gordon [5,6] tried to describe dielectric media by an "effective metric" and suggested using a gravitational field to mimic a dielectric medium. Interestingly, in contrast to Gordon [5], Landau and Lifshitz [7] tried to use dielectric media to simulate a gravitational field.

In connection with mapping of EM fields in physical space to the electromagnetism of empty flat space, let note the paper [8] dating back to 1959 where the covariant Maxwell equations [9] in a non-inertial reference system were transformed to the usual three dimensional vector form for the particular problem of EM field study in a rotating system of reference. In this case the displacement vector $\vec{D}$ and the magnetic field vector $\vec{B}$ were expressed through the electric field vector $\vec{E}$ and the magnetic flux vector $\vec{H}$ via the constitute equations

$$
\begin{aligned}
\vec{D} & =\vec{E}-(1 / c) \vec{v} \times \vec{H}, \\
\vec{B} & =\vec{H}+(1 / c) \vec{v} \times \vec{E}
\end{aligned}
$$

where $\vec{v}$ was a local speed of rotating. The formulas were also obtained relating the electric field and the magnetic flux in a non-inertial and an inertial systems of reference. In 1960 Plebanski [10] formulated the EM effect of the curved space-time or curved coordinates in concise constitutive equations for a general case. The constitutive equations [10] are obtained from the abovementioned ones by replacing the first terms in the right hand side of these equations with $\varepsilon \vec{E}$ and $\mu \vec{H}$, respectively, and by replacing the vector $\vec{v}$ with $-\overrightarrow{\boldsymbol{\omega}}$, where the symmetric matrices $\varepsilon=\mu$ and vector $\overrightarrow{\boldsymbol{\omega}}$ are written in terms of the space-time metric tensor. Now after Pendry et al [1], media with such $\varepsilon$ and $\mu$ and $\vec{\omega}$ are called the transformation media. The transformation media lead to modern metamaterials that include the so-called left- 
handed materials (LHMs), which demonstrate not only the superlensing effect but are able to avoid objects and flow around them, as it was demonstrated numerically [1] and analytically in terms of bistatic scattering [11]. A flat perfect LHM slab is commonly referred to as Veselago's lens [12] in which both the permittivity $\varepsilon$ and the permeability $\mu$ are equal to minus one. The terms optical left- and righthandedness were introduced by Veselago [12] to distinguish LHMs, in which the wave vector, the electric and magnetic fields vectors of a wave form a left-handed orthogonal set from conventional right-handed media with the right-handed triple of the same vectors.

The superlensing effect was demonstrated experimentally with a slab of silver [13] and perovskites [14]. Resonantly coupled plasmon polaritons propagating on both surfaces of the silver slab were identified to be responsible for the superlensing effect. The slab volume itself seems not so important. Schweizer et al [15] supposed that resonantly coupled frequency selective surfaces, e.g. stacks of multiple meander structures, might also cause subwavelength imaging. Geometrical parameters of the meander structures (corrugation and spacer between metal films) may be changeable to a large degree.

In this paper we present a phenomenon of virtual singular scattering of an electromagnetic wave on an inhomogeneity located in the volume of a left - handed material slab using a less known property of the left-handed material slab to transfer the singularity of the free space Green function through implementing coordinate transformation.

We use the Green function approach [16] elaborated for a line source wave scattering by the line dielectric inhomogeneity inside the LHM slab. The central point of our method is a transformed integral equation for the Green function of an inhomogeneous LHM slab written in terms of the Green function for a homogeneous LHM slab and a volume scattering potential accounting for the dielectric inhomogeneity inside the slab. The solution to this transformed integral equation is presented with the aid of a scattering operator (T-matrix), which satisfies the LippmanSchwinger equation. The solution to the Green function for an inhomogeneous LHM slab is expressed by a scattering amplitude in the case of a linelike scatterer which is thin as compared with the free space wavelength. The scattering amplitude was evaluated through an exact solution to the Lippman - Schwinger equation via modeling the scattering potential of the linelike scatterer by a non-local separable potential.

\section{Scattering operator}

Let s-polarized monochromatic EM wave with the frequency $\omega$ be incident from the background medium $\Omega_{0}$ with both permittivity and permeability equal to unity, $\varepsilon_{0}=\mu_{0}=1$, onto an $L$ thick 2D inhomogeneous LHM slab (region $\Omega_{1}$ bounded from two sides by the planes $z=$
0 and $z=L$ of the Cartesian coordinate system $x, y, z)$. The electric field of the wave has only the $y$-component $E_{y}(x, z)=\left(4 \pi \omega \mu_{0} / i c^{2}\right) G j_{y}$. Here operator denotations and the Gaussian systems of units are used; $G\left(\vec{\rho}, \vec{\rho}^{\prime}\right)$ is the 2D Green function for the LHM slab, $j_{y}$ stands for the current density of the EM field source. The points $\vec{\rho}=(x, z)$ and $\vec{\rho}^{\prime}=\left(x^{\prime}, z^{\prime}\right)$ are placed inside the regions $\Omega_{a}$ and $\Omega_{b}$, respectively, where indices $a, b=$ 0,1 .

We describe LHM using the dielectric permittivity and the magnetic permeability which are both effective-medium parameters of artificial composite materials. In the case of natural substances, the magnetic permeability ceases to have any physical meaning already at relatively low frequency [17]. However, unlike natural substances, artificial composite materials involving substances with exceedingly large dielectric permittivity may nevertheless show a magnetic response which is incompatible with the Landau-Lifshitz argument [18].

An inhomogeneous LHM slab means a spatially regular LHM slab which possesses nonrandom inhomogeneities consisting of right-handed nonmagnetic material. The inhomogeneous slab has an inhomogeneous dielectric permittivity $\varepsilon(x, z)=-1+\delta \varepsilon_{0}+\delta \varepsilon(x, z)$ and homogeneous magnetic permeability $\mu=\mu^{\prime}+i \mu^{\prime \prime}$ whose real part $\mu^{\prime}$ can be close to minus one and $\mu^{\prime \prime} \rightarrow 0$. The homogeneous deviation $\delta \varepsilon_{0}$ of the dielectric permittivity and the small imaginary part $\mu^{\prime \prime}$ of magnetic permeability both save the convergence of the Green function $G^{(0)}\left(\vec{\rho}, \vec{\rho}^{\prime}\right)$ of a homogeneous slab for any receiver and source plane positions (see section 2.1). The nonrandom part $\delta \varepsilon(x, z)$ accounts for inhomogeneities. Clearly, the limit $\delta \varepsilon(x, z) \rightarrow 0$ corresponds to vanishing of inhomogeneities and, hence, the situation of a homogeneous LHM slab.

The Green function $G\left(\vec{\rho}, \vec{\rho}^{\prime}\right)$ is naturally subdivided into four pieces [16] $G_{a, b}\left(\vec{\rho}, \vec{\rho}^{\prime}\right)$ depending on that in which region $\Omega_{a, b}$ is positioned, the source $\vec{\rho}^{\prime}$ and the receiver $\vec{\rho}$ points. Physically $G_{00}$ describes wave radiation transmitted through and reflected from the slab, $G_{10}$ and $G_{01}$ describe radiation incoming into and outgoing from the slab, respectively, and $G_{11}$ describes radiation propagated inside the slab. These four Green functions are linked to each other by the standard boundary conditions of the electric and magnetic field tangential component continuity to the slab boundaries.

The extended boundary condition technique [19] allowed [16] to derive specific radiation conditions on the slab boundaries for the Green function $G_{10}$ of incoming 
radiation and the integral equation for this Green function with an effective scattering potential followed by a continuous extension of $G_{10}$ from the slab boundaries to the slab outside region, which gives the Green function $G_{00}$. Similarly, specific radiation conditions on the slab boundaries can be derived for the Green function $G_{11}$ of propagating radiation together with the integral equation for this Green function with an effective scattering potential followed by a continuous extension of $G_{11}$ from the slab boundaries to the slab outside region, which gives the Green function $G_{01}$.

Then we write down the Green function of an inhomogeneous LHM slab $G_{a b}$ in terms of the Green function of a homogeneous one $G_{a b}^{(0)}$. In particular, we have

$$
G_{00}=G_{00}^{(0)}+G_{01}^{(0)} T G_{10}
$$

where the scattering operator of the inhomogeneity $T\left(\vec{\rho}, \vec{\rho}^{\prime}\right)$ obeys the Lippman - Schwinger equation

$$
T=V_{1}+V_{1} G_{11}^{(0)} T
$$

Here $V_{1}(x, z)=-k_{0}^{2}[\delta \varepsilon(x, z)] \mu$ is the inhomogeneous component of the effective volume scattering potential $V(x, z)=V_{0}+V_{1}(x, z) \quad$ which also has the homogeneous $V_{0}=k_{0}^{2}-k_{01}^{2}$ part; $k_{0}=\omega / c$ and $k_{01}=k_{0}\left[\left(-1+\delta \varepsilon_{0}\right) \mu\right]^{1 / 2}$ are the wave numbers in the free space and homogeneous LHM slab, respectively. The effective volume scattering potential $V(x, z)$ has the 1D Fourier transform with respect to the $x$-component of the spatial position vector

$$
\hat{V}\left(q, q^{\prime}, z\right)=\hat{V}_{0}\left(q, q^{\prime}, z\right)+V_{1}\left(q-q^{\prime}, z\right)
$$

with a singular potential of a homogeneous slab

$$
\hat{V}_{0}\left(q, q^{\prime}, z\right)=V_{0} 2 \pi \delta\left(q-q^{\prime}\right)+V_{S}\left(q, q^{\prime}, z\right)
$$

and a regular volume potential $V_{1}\left(q-q^{\prime}, z\right)$ accounting for inhomogeneities. The singular surface part

$$
\begin{aligned}
& V_{S}\left(q, q^{\prime}, z\right)= \\
& (1-\mu) i \gamma_{q}[\delta(z-\eta)+\delta(z-L+\eta)] 2 \pi \delta\left(q-q^{\prime}\right)
\end{aligned}
$$

describes the effect of a magnetic permeability jump on the slab boundaries, $\eta \rightarrow 0 ; \gamma_{q}=\left(k_{0}^{2}-q^{2}\right)^{1 / 2}$ stands for the longitudinal wave number.

\subsection{Green function $G_{a b}^{(0)}$ singularity transfer by homogeneous LHM slab}

In the case of a homogeneous slab $(\varepsilon=\mu=-1)$ the effective potential $\hat{V}\left(q, q^{\prime}, z\right)$ consists only of surface part $V_{S}\left(q, q^{\prime}, z\right)$ which simplifies the abovementioned integral equations for the Green functions $G_{10}$ and $G_{11}$ to an algebraic and easily resolved form. The outgoing radiation Green function $G_{01}$ can be obtained on the basis of a reciprocity relation [16] $\left(1 / \mu_{a}\right) G_{a, b}\left(\vec{\rho}, \vec{\rho}^{\prime}\right)=$ $\left(1 / \mu_{b}\right) G_{b, a}\left(\vec{\rho}^{\prime}, \vec{\rho}\right)$.

Figure 1 demonstrates that the Green function $G^{(0)}\left(\vec{\rho}, \vec{\rho}^{\prime}\right)$ of perfect Veselago's lens is simply obtained $[2,16]$ from the empty space Green function $G_{0}\left(\vec{\rho}-\vec{\rho}^{\prime}\right)$ by a spatial coordinate transformation from the physical $z$ axis to the EM (empty) space $\widetilde{z}$ coordinate: $\widetilde{z}=z$ if $z<0, \widetilde{z}=-z$ if $0<z<L$, and $\widetilde{z}=z-2 L$ if $z>L$. However, these relations are only valid for definite

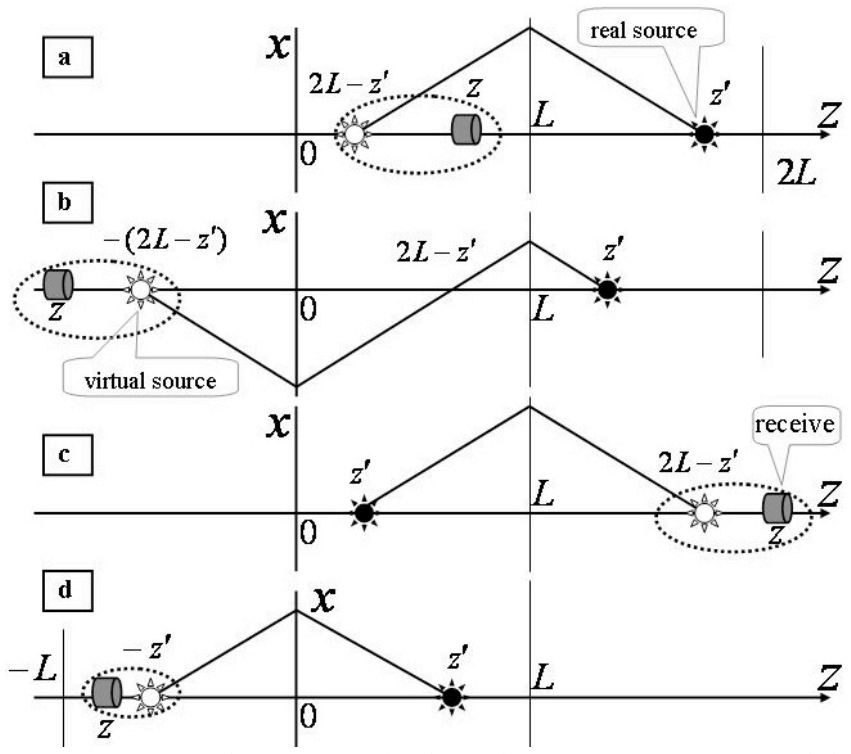

Figure 1: Singularity transfer by a homogeneous LHM slab from the right outside plane $z=z^{\prime}, L<z^{\prime}<2 L$, to the inside (a) and left outside (b) focal planes $z \rightarrow 2 L-z^{\prime}$, $z>2 L-z^{\prime}$ and $z \rightarrow-\left(2 L-z^{\prime}\right), \quad z<-\left(2 L-z^{\prime}\right)$, respectively; the singularity transfer from the inside plane $z=z^{\prime}, 0<z^{\prime}<L$ to the right (c) and left (d) outside focal planes $z=2 L-z^{\prime}, \quad z>2 L-z^{\prime}$ and $z \rightarrow-z^{\prime}$, $z<-z^{\prime}$, respectively.

positions of receive and source planes. Otherwise, the Green function $G^{(0)}$ in an angular spectrum representation is diverged due to the evanescent wave enhancement effect [20]. Really, Figure 1(a) schematically shows that incoming radiation Green function $G_{10}^{(0)}$ coincides with the empty space Green function $G_{10}^{(0)}=G_{0}\left(x-x^{\prime}, z-2 L+z^{\prime}\right)=$ $G_{0}\left(x-x^{\prime},-\widetilde{z}+\widetilde{z}\right)$ and has the singularity at the inside focal plane $z \rightarrow 2 L-z^{\prime}$ under the conditions $z>2 L-z^{\prime}$ and $L<z^{\prime}<2 L$. The other three panels of Figure $1(b, c, d)$ illustrate the properties of the transmitted radiation Green function (panel b) $G_{00}^{(0)}=$ 
$G_{0}\left(x-x^{\prime},-\widetilde{z}+\widetilde{z}^{\prime}\right)$ with the singularity at the left outside focal plane $z \rightarrow-\left(2 L-z^{\prime}\right)$ under the conditions $z<-\left(2 L-z^{\prime}\right)$ and $L<z^{\prime}<2 L$; the Green function of radiation outgoing from inside to the right outside slab region (panel c) $G_{01}^{(0)}=-G_{0}\left(x-x^{\prime}, \widetilde{z}-\widetilde{z}^{\prime}\right)$ with the singularity at the right outside focal plane $z \rightarrow 2 L-z^{\prime}$ under the condition $z>2 L-z^{\prime}$; and the Green function of radiation outgoing from inside to the left outside slab region (panel d) $G_{01}^{(0)}=-G_{0}\left(x-x^{\prime}, \widetilde{z}^{\prime}-\widetilde{z}\right.$ ) with the singularity at the left outside focal plane $z \rightarrow-z^{\prime}$ under the condition $z<-z^{\prime}$.

Figure 1(a), for example, can be treated as if source and receiver are situated in the positions $2 L-z^{\prime}$ and $z$, constant potential to be $V_{1}=k_{0}^{2} \delta \varepsilon$, we replace the unknown Green function $G_{10}$ in Eq.(1) with a known one $G_{10}^{(0)}$ in a perfect Veselago's lens that gives an approximate equation

$$
G_{00} \approx G_{00}^{(0)}+G_{01}^{(0)} T G_{10}^{(0)}
$$

For definiteness we consider below the case of a small linelike inhomogeneity infinitely extended along the $y$ axis (see black box in Figure 2) and whose rectangular cross section occupies a region in the $x z$-plane with the centre point $x_{1}, z_{1}$ and linear dimensions $\Delta x, \Delta z$. Assuming these dimensions to be smaller than the wavelength in the free space, $k_{0} \Delta x<<1$ and $k_{0} \Delta z<<1$, Eq.(3) reads

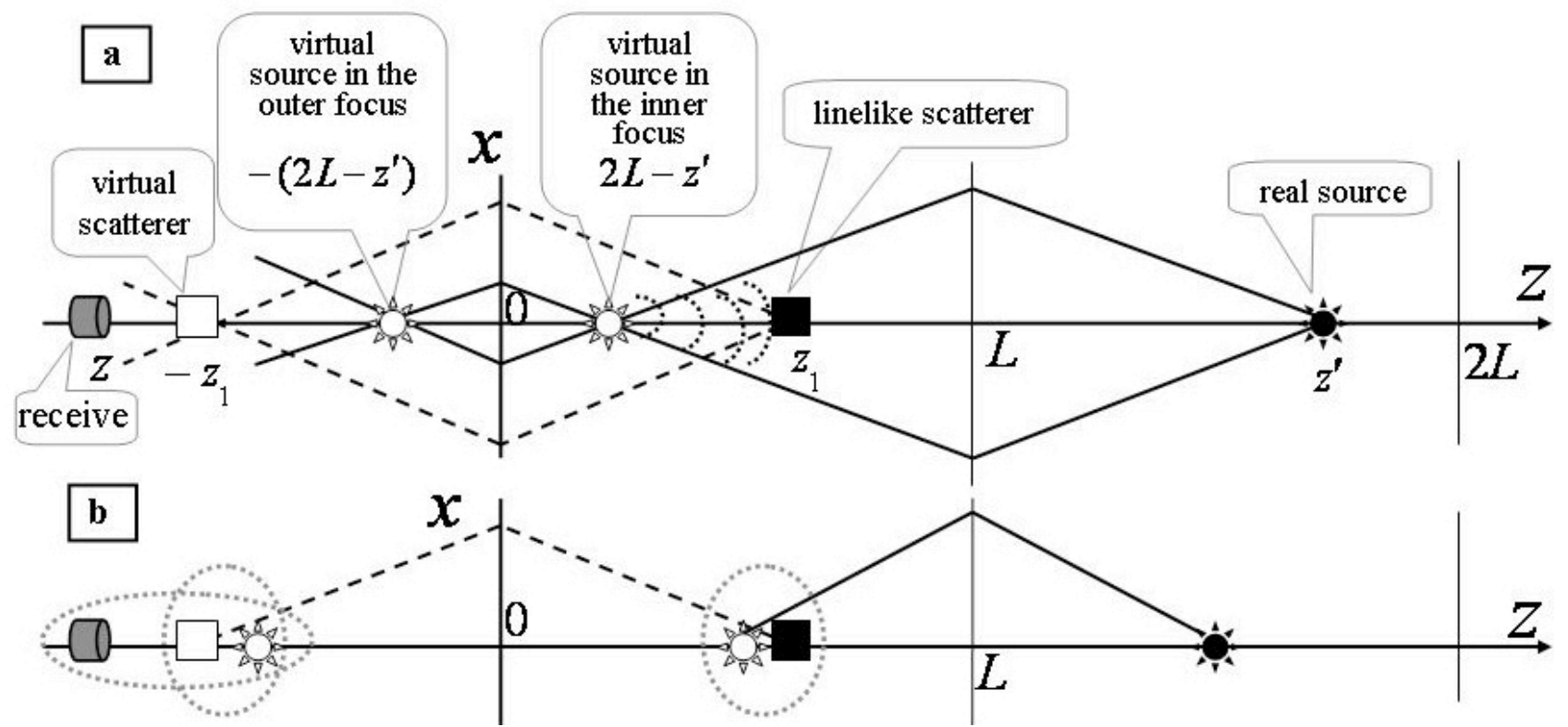

Figure 2: (a) Multiplicative effect in singularity transfer from the right outside plane $z=z^{\prime}$ at some position $0<z^{\prime}<2 L$ to the left outside focal plane, $z=-\left(2 L-z^{\prime}\right)$, by LHM slab via linelike scatterer $z_{1}$. (b) At a definite position of the real source $z^{\prime}$, the real $z_{1} \rightarrow 2 L-z^{\prime}, z_{1}>2 L-z^{\prime}$ and virtual $z<-z_{1}<-\left(2 L-z^{\prime}\right), z \rightarrow-\left(2 L-z^{\prime}\right)$ scatterers are radiated by singular quasistatic fields of inside $2 L-z^{\prime}$ and outside $-\left(2 L-z^{\prime}\right)$ virtual sources, respectively, and receive is in the near field region of both outside virtual source and virtual scatterer.

respectively. Besides, note that the inside slab source image can be obtained on the outside region of the slab in the last two panels of Figure 1(c,d).

\section{Scattering amplitude}

Physically Eq.(1) evidently means that radiation created by, e.g., a point source on the right outside slab region and then transmitted through or reflected from the inhomogeneous LHM slab is the radiation incoming into the slab, scattered by that slab volume inhomogeneities and then outgoing from the slab. A detailed investigation of the outgoing radiation has been precluded by that the Green function $G_{10}$ for radiation incoming into an inhomogeneous slab is unknown. Therefore, assuming a weak scatterer

$$
\begin{aligned}
& G_{00}\left(x, z ; x^{\prime}, z^{\prime}\right) \approx G_{00}^{(0)}\left(x, z ; x^{\prime}, z^{\prime}\right) \\
& +\widetilde{T} G_{01}^{(0)}\left(x, z ; x_{1}, z_{1}\right) G_{10}^{(0)}\left(x_{1}, z_{1} ; x^{\prime}, z^{\prime}\right)
\end{aligned}
$$

Here $\widetilde{T}$ denotes a scattering amplitude that is obtained by integrating the scattering operator Eq.(2) over all its four arguments. Physically Eq.(4) represents a multiplicative effect in the singularity transfer beyond the Born approximation written in [16] as $G_{00} \approx G_{00}^{(0)}+V_{1} G_{01}^{(0)} G_{10}^{(0)}$.

Figure 2(a) schematically demonstrates this effect. It is clear that the real source can be freely moved in the slab outside region $L<z^{\prime}<2 L$. A current position of the real source defines current positions of the inner and outer slab 
focuses. At a definite position of the real source, the real and virtual scatterers are radiated by singular quasistatic fields of inside and outside virtual sources, respectively. Besides, a receiver is situated in the near field region of both outside virtual source and virtual scatterer (dotted lines in Figure 2(b)).

\section{Multiplicative effect in singularity transfer}

\subsection{Transmission mode}

Let us introduce the physically important defocusing parameters $\delta z$ and $\delta z^{\prime}$ for the positions of receiver and source planes, respectively, relatively to the position $z_{1}$ of a linelike scatterer plane defined by $\delta z=-z-z_{1}$ and $\delta z^{\prime}=z_{1}-2 L+z^{\prime}$. Supposing these defocusing parameters are much larger than the linelike scatterer cross- $\delta z=z-2 L+z_{1}$ and $\delta z^{\prime}=z^{\prime}-2 L+z_{1}$ which gives (Fig.3(b))

$$
\begin{aligned}
& G_{00}\left(x, z ; x^{\prime}, z^{\prime}\right) \approx G_{00}^{(0)}\left(x=0, z-z^{\prime}\right) \\
& -\widetilde{T} G_{0}(x=0, \delta z) G_{0}\left(x=0, \delta z^{\prime}\right)
\end{aligned}
$$

\section{Exact scattering amplitude under resonance}

The last step is to demonstrate that a small inhomogeneity in the LHM slab can significantly contribute into the scattered field in the regime similar to the well known Mie resonance scattering. Following [21], let us replace the local scattering potential $V_{1}(x, z)$ in Eq.(2) with an auxiliary non-local separable scattering potential $V_{1}(x, z) \delta\left(x-x^{\prime}\right) \delta\left(z-z^{\prime}\right) \rightarrow f_{1} \xi(x, z) \xi\left(x^{\prime}, z^{\prime}\right)$
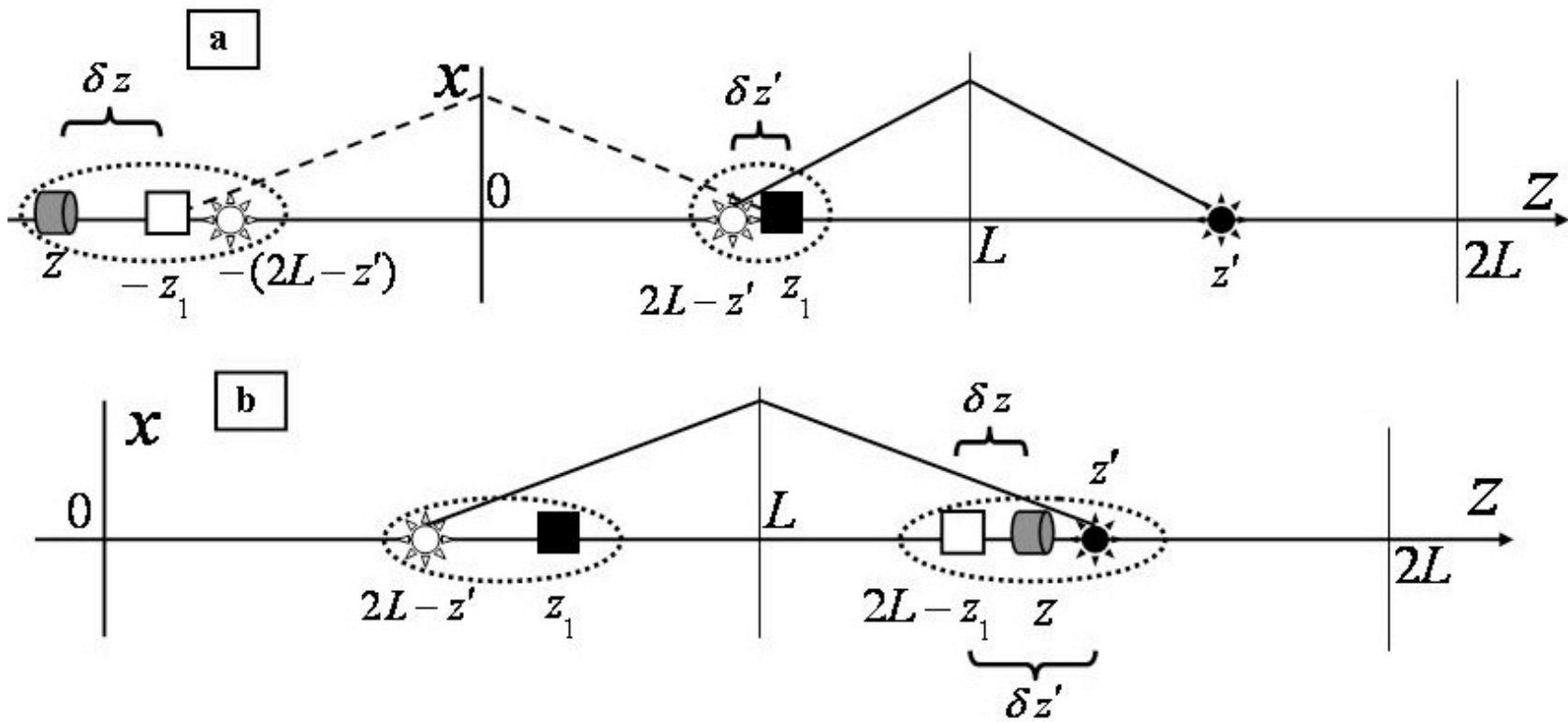

Figure 3: Multiplicative effect in singularity transfer by LHM slab from the right outside plane $z=z^{\prime}$ to the left (a) and right (b) outside focal planes $z \rightarrow-\left(2 L-z^{\prime}\right), z<-z_{1}<-\left(2 L-z^{\prime}\right)$, and $z \rightarrow 2 L-z_{1}, z^{\prime}>z>2 L-z_{1}$, respectively, via linelike scatterer $z=z_{1}$ near inside focal plane $z_{1} \rightarrow 2 L-z^{\prime}, z_{1}>2 L-z^{\prime}$.

section dimensions, $\delta z>\Delta x, \Delta z$ and $\delta z^{\prime}>>\Delta x, \Delta z$, and an exact focusing with respect to the $x$ axis, $x=x^{\prime}=x_{1}$, Eq.(4) can be simplified as (Fig.3(a))

$$
\begin{aligned}
& G_{00}\left(x, z ; x^{\prime}, z^{\prime}\right) \approx G_{00}^{(0)}\left(x=0,-z-2 L+z^{\prime}\right) \\
& -\widetilde{T} G_{0}(x=0, \delta z) G_{0}\left(x=0, \delta z^{\prime}\right)
\end{aligned}
$$

\subsection{Reflected mode}

Simplification of Eq.(4) in the case of slab reflected radiation, with the receiver and source planes being placed both in the right $z>L$ and $z^{\prime}>L$ outside region of the slab, is performed similarly to the case of transmitted radiation: the defocusing parameters are now defined by
[16] and the function $\xi(x, z)$ is normalized to unity. The Lippman -Schwinger Eq.(2) with a separable potential is resolved exactly in accordance with the well known theory of potential scattering [22]. The corresponding solution for the scattering amplitude gives

$$
\widetilde{T}=\frac{f_{1}}{1-f_{1}\left(\xi G_{11}^{(0)} \xi\right)}
$$

where a bilinear form with a scalar product is in the denominator.

The Green function $G_{11}^{(0)}$ of radiation propagating inside a perfect planar LHM slab is presented by the divergent integral [16]. However this Green function has been numerically calculated [23] for a lossy slab with 
$\varepsilon=-1+i \delta_{\varepsilon}$ and $\mu=-1+i \delta_{\mu}$ where both $\delta_{\varepsilon}$ and $\delta_{\mu}$ are small positive real numbers. We performed an analytical estimation of this Green function in the asymptotical limit $\mu^{\prime}=-1, \mu^{\prime \prime} \rightarrow 0$ and revealed a resonance property of the scattering amplitude (8) with its resonance value

$$
\widetilde{T} \approx f_{1} / i \mu^{\prime \prime}
$$

under the resonance condition

$$
k_{0} \Delta z \approx\left(\frac{\Delta z}{L}\right)^{2}\left(\ln \frac{1}{\left|\mu^{\prime \prime}\right|}\right)^{2}\left(\frac{\left|\mu^{\prime \prime}\right|}{\delta \varepsilon}\right)^{1 / 2} \ll 1
$$

where both dimensions of the scatterer cross section are equal to $\Delta x=\Delta z$. Fig.4 demonstrates that the resonance value of the scattering amplitude (9) may be not so small for inhomogeneity dimensions of practical interest. At the same time, the inset in Fig. 4 proves that the resonance condition (10) is satisfied, i.e. that the scatterer cross section has to be

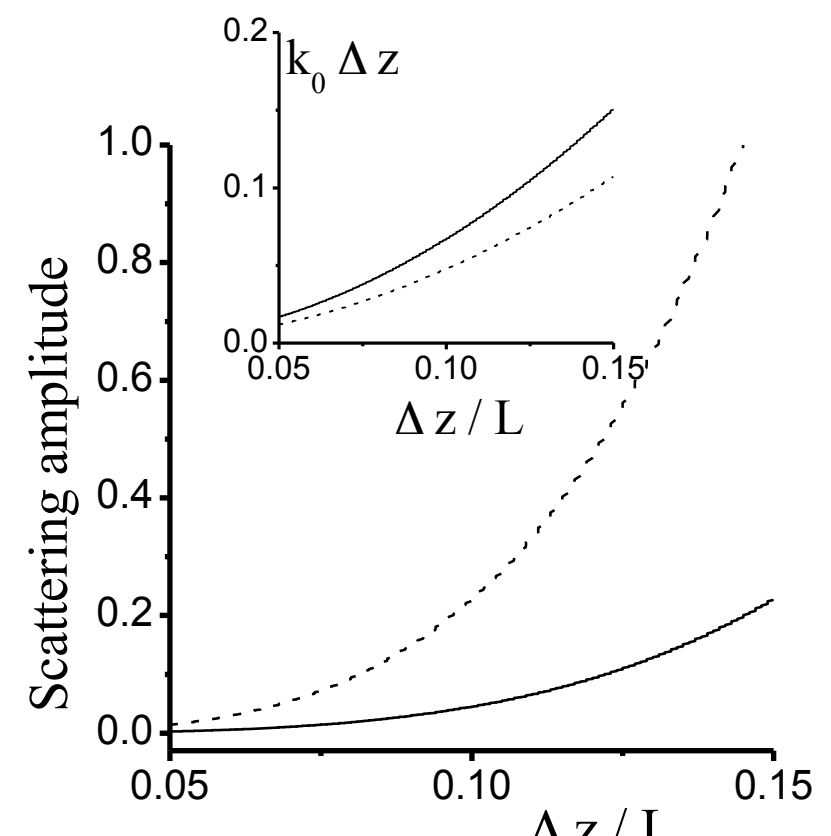

Figure 4: Calculated resonance scattering amplitude (9) versus the normalized dimension of the linelike inhomogeneity with small constant value of the dielectric permittivity $\delta \varepsilon=0.1$ inside the LHM slab (black box in Figure 2) for the imaginary part of the inhomogeneity magnetic permeability $\mu^{\prime \prime}=10^{-2}$ (solid curves), $10^{-3}$ (dashed curves) and the magnetic permeability real part equal to $\mu^{\prime}=-1$. The inset visualizes the inequality (10).

smaller than the free space wavelength.

\section{Discussion}

Let us discuss the resolution of a linelike scatterer with respect to its depth extension in the LHM slab. Firstly note that the defocusing parameters $\delta z$ and $\delta z^{\prime}$ defined in the section 4 are supposed to be much larger than the linelike scatterer cross dimensions. Nevertheless, these defocusing parameters can be much less than the wave number in the background, $k_{0} \delta z<<1$ and $k_{0} \delta z^{\prime}<<1$, until the linelike scatterer cross dimensions are also much less than the wave number, $k_{0} \Delta z<<1$ and $k_{0} \Delta z^{\prime}<<1$.

Consider in greater detail Eq.(6) for the Green function of inhomogeneous slab reflected radiation

$$
\begin{aligned}
G_{00}\left(x, z ; x^{\prime}, z^{\prime}\right) & \approx G_{00}^{(0)}\left(x=0, z-z^{\prime}\right) \\
& +G_{00}^{(R)}\left(x_{1}, z ; x_{1}, z^{\prime}\right)
\end{aligned}
$$

where the term $G_{00}^{(R)}=-\widetilde{T} G_{0}(x=0, \delta z) G_{0}\left(x=0, \delta z^{\prime}\right)$ takes the form

$$
G_{00}^{(R)}\left(x_{1}, z ; x_{1}, z^{\prime}\right) \approx-\widetilde{T} \frac{1}{(2 \pi)^{2}}\left(\ln \frac{2}{k_{0} \delta z}\right)\left(\ln \frac{2}{k_{0} \delta z^{\prime}}\right)
$$

in the quasistatic limit. We remind that the empty space Green function is proportional to the first type Hankel function of the zero order, $G_{0}(\vec{\rho})=(1 / 4 i) H_{(0)}^{1}\left(k_{0} \vec{\rho}\right)$, and asymptotically tends to the logarithm expression, $(1 / 2 \pi) \operatorname{Ln}\left(2 / k_{0} \vec{\rho}\right)$, in the quasistatic limit, $k_{0} \vec{\rho}<<1$.

The term $G_{00}^{(R)}$ describes the contribution of a linelike scatterer into the Green function of the inhomogeneous LHM slab in the case of reflection from the slab. This term is proportional to the scattering amplitude of the scatterer and is the product of two Green functions' quasistatic singularities, provided the source and receiver points being focused on the scatterer with small defocusing parameters.

A resolution of a linelike scatterer with respect to its depth extension is defined by the first logarithm in the right hand side of Eq.(12). Fig.5 gives illustration to Eq.(12) presenting the normalized quantity $G_{00}^{(R)}$ as a function of the defocusing parameter $\delta z$ measured in the linelike scatterer thickness $\Delta z$.

\section{Conclusions}

We describe a phenomenon of electromagnetic wave virtual singular scattering as if the wave virtual receiver and virtual source points were in the near zone of a scatterer. Such singular scattering can be realized in the case when the scatterer is positioned inside a left-handed material slab and the real source and real receiver points are placed at definite positions outside the slab.

Let us stress that a phenomenon of virtual singular scattering is inherent exclusively in such artificial media which transfer the singularity of the free space Green function by implementing the coordinate transformation. Contrary, 'ordinary' singular scattering can be realized in natural media. The paper [24] gives interesting example for real (not virtual) singular scattering when a scatterer and an observation point (receive) both approach the so-called near field zone of a source of electromagnetic waves and the scattering process becomes singular via the spatial singularity of the free space Green function at the origin. In the paper [24] such real singular scattering situation is 
realized in the framework of the moving ionospheric ridge employing to develop a method treating the inverse scattering problem for a buried inhomogeneous dielectric strip, based on ground field measurements. The real singular scattering situation is considered in [24] with the aid of total electric field evaluation inside the scatterer volume that forced to make restriction of Born approximation. In difference from [24], we apply a more productive technique of $\mathrm{T}$ - scattering operator of inhomogeneity obeying the Lippman - Schwinger equation to s-polarized electric wave field multiple scattering by a dielectric inhomogeneity placed inside a left handed material slab. This technique enables us to consider Mie resonances of inhomogeneity in situation of virtual (not real) singular scattering.

As is known [25], light distribution near the focus of a right handed material lens is written in terms of Lommel's functions that are regular and non-singular. Contrary, the wave field near the inside focus of a perfect Veselago's lens is described by the Green function of empty space, with the virtual source point being placed in this inside focus. The wave field near an outside perfect Veselago' s lens focus also demonstrates a similar singular behaviour and can be

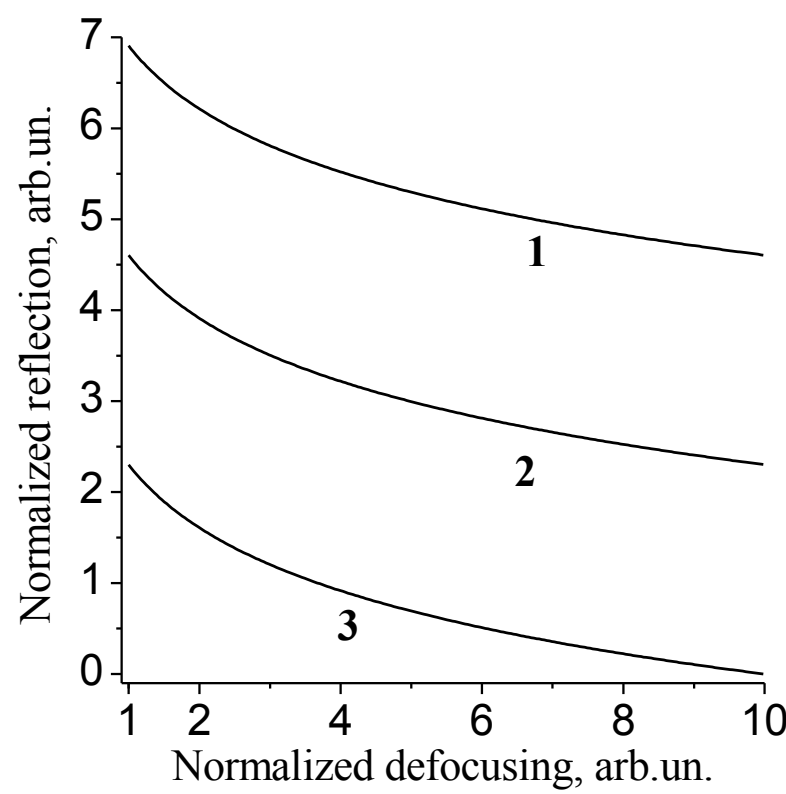

Figure 5: The dependence of the normalized reflection $G_{00}^{(R)}\left[-\widetilde{T}(2 \pi)^{-2} \ln \left(2 / k_{0} \delta z^{\prime}\right)\right]^{-1}$ from linelike scatterer on the normalized defocusing parameter $\delta z / \Delta z$ at different values of the parameter $k_{0} \Delta z / 2=10^{-3}$ (curve 1), 0.01 (2), and 0.1 (3).

described by the empty space Green function with a virtual source point placed in this outside focus. As a consequence, the result is the phenomenon of singular wave scattering by a weak and thin dielectric inhomogeneity as a linelike scatterer placed near the inside focus of Veselago's lens. In this case the linelike scatterer is radiated by a singular quasistatic field near the inner focus of Veselago's lens that enhances the effect of scattering. Besides, the scattered radiation outgoing from the left-handed material slab is enhanced in a similar singular manner near the outside focus of Veselago's lens. As a result, the effect of wave scattering by a linelike scatterer near the inside Veselago's lens focus appears to be proportional to the product of singular quasistatic parts of two empty space Green functions, which means a multiplicative quasistatic singularity of the Green function for an inhomogeneous lefthanded material slab.

Modelling the linelike scatterer by a non-local separable scattering potential reveals a resonance property of the scattering amplitude related to the singular behaviour of the Green function for waves propagating inside a perfect lefthanded material slab.

Finally, note that the positions of the inside and outside focuses of a flat slab of homogeneous left handed material (perfect Veselago' s lens) are defined by the positions of a real source and a real receiver points and a slab thickness. Therefore for a given small inhomogeneity inside a lefthanded material slab such positions of real source and real receiver points can be found which gives rise to a visible image of the inhomogeneity due to the described phenomenon of singular scattering.

The latter statement has at least two conclusions. First, the principle of non-contact tunneling near field optical microscopy with some features of tomography can be proposed using optical singularities produced by a Veselago like medium, in a manner as if an optical antenna of a near field scanning microscope would be able to penetrate inside a tested surface [26]. Indeed, Veselago like medium allows the displaying of a set of near field images taken from different observation points of an object using any projection rendering system. Due to the phenomenon of electromagnetic wave virtual singular scattering, the brightness of an object image would be as high as if the source and receiver were both located in the near field zone of the object. Second, in the Introduction we note that lefthanded materials are currently considered as media which enable precise control over the flow of electromagnetic waves. Using these artificial materials, the first microwave cloaking has been achieved [27]. However, even an ideal perfect cloak (curved electromagnetic space) may be electromagnetically detected within its working band [28] on the basis of radiation (some kind of electromagnetic source) generated at the propagation of a fast-moving charged particle through the cloak. At the same time, perfect artificial material consisting of huge amounts of identical electrotechnical elements are rather an illusion in practice. Defects of such materials may function as the abovementioned current sources [28]. One sort of such defects, i.e. a dielectric inhomogeneity in a left handed material slab, has been considered in this paper.

\section{Acknowledgements}

This work was supported in part by the Russian Academy of Sciences projects "Passive multichannel human radio- and acusto-thermotomography in near zone", "Scientific bases of heterogeneous telecommunication and location systems". 


\section{References}

[1] J.B. Pendry, D. Schurig, D.R. Smith, Controlling electromagnetic fields, Science 312: 1780- , 2006.

[2] U. Leonardt, T.G. Philbin, General relativity in electrical engineering, New J. Phys. 8: 247- , 2006.

[3] U. Leonhardt, Optical conformal mapping, Science 312: 1777- 1780, 2006.

[4] F. de Felice, On the gravitational field acting as an optical medium, General Relativity and Gravitation 2: 347-357, 1971.

[5] W. Gordon, Zur lichtfortpflanzung nach der relativitätstheorie, Ann. Phys. (Leipzig) 72: 421-456, 1923.

[6] S. Antoci, L. Mihich, A forgotten argument by Gordon uniquely selects Abraham's tensor as the energymomentum tensor for the electromagnetic field in homogeneous, isotropic matter, http://arxiv.org/abs/gr$q c / 9704055 v 1$

[7] L.D. Landau, E.M. Lifshitz, The Classical Theory of Fields, Fourth Revised English Edition, Pergamon, Oxford, UK, p. 275, 1975.

[8] Yu.N. Barabanenkov, Maxwell equations in rotating system of reference, Scientific Reports of Higher School, Phys-Math Sciences 1: 141 - 145, 1959.

[9] A. Einstein, Die Grundlage der allgemeinen Relativitätstheorie, Ann. Phys. 49: 769-822 , 1916; translated without p.769 as The Foundation of the General Theory of Relativity, The Principle of Relativity, Dover, New York, pp. 111-164,1952.

[10] J. Plebanski, Electromagnetic waves in gravitational fields, Phys. Rev. 118: 1396 - 1408, 1960.

[11] H. Chen, Bae-Ian Wu, B.Zhang, J.A. Kong, Electromagnetic wave interactions with a metamaterial Cloak, Phys. Rev. Lett. 99: 063903 (2007)

[12] V.G. Veselago, The electrodynamics of substances with simultaneously negative values of $\varepsilon$ and $\mu$, Sov. Phys. Usp. 10: 509-514, 1968.

[13] N. Fang, H. Lee, Ch. Sun, X. Zhang, Sub-diffractionlimited optical imaging with a silver superlens, Science 308: 534-537, 2005.

[14] S.C. Kehr, Y.M. Liu, L.W. Martin, P. Yu, M. Gajek, S.-Y. Yang, C.-H. Yang, M.T. Wenzel, R. Jacob, H.-G. von Ribbeck, M. Helm, X. Zhang, L.M. Eng, R. Ramesh, Near-field examination of perovskite-based superlenses and superlens-enhanced probe-object coupling, Nature Communications 249: 1-9, 2011

[15] P. Schau, K. Frennera, L. Fub, H. Schweizer, H. Giessenb, W. Osten, Rigorous modeling of meandertype metamaterials for sub-lambda imaging, Proc. SPIE 8083: 808303, 2011

[16] Yu.N. Barabanenkov, M.Yu. Barabanenkov, S.A. Nikitov, Line source wave scattering by line inhomogeneities inside left-handed material slab: Green function approach, Proc. PIERS Cambridge, USA, pp.789-799, 2008.
[17] L.D. Landau, E.M. Lifshitz, Electrodynamics of Continuous Media, Pergamon, Oxford, UK, p. 251, 1981.

[18] R. Merlin, Metamaterials and Landau-Lifshitz permeability arguments: large permittivity begets highfrequency magnetism, PNAS:106, 1693-1698, 2009.

[19] L. Tsang, J.A. Kong, R. Shin, Theory of Microwave Remote Sensing, John Wiley, New York, 1985.

[20] B. Pendry, Negative refraction makes a perfect lens, Phys. Rev. Lett. 85: 3966 - 3969, 2000

[21] Yu.N. Barabanenkov, M.Yu. Barabanenkov, Radiative transfer theory with time delay for effect of a pulse imprisonment in a resonant random media: general transfer equation and point-like scatterer model, Waves in Random Media 7: 607-633 ,1997.

[22] J.R. Taylor, Scattering Theory: the Quantum Theory of Nonrelativistic Collisions, John Wiley, New York, 1972.

[23] Pi-G. Luan, H.-Da Chien, Ch.-Ch. Chen, Chi-Sh. Tang, Analysis on the imaging properties of a lefthanded material slab, arXiv:physics/0311122v2 [physics.optics]

[24] C.A. Valagiannopoulos, N.K. Uzunoglu, Simplified model for EM inverse scattering by longitudinal subterranean inhomogeneities exploiting the dawn/dusk ionospheric ridge, IET-Micro. Antennas Propag. 5: $1319-1327,2011$

[25] M. Born, E. Wolf. Principles of Optics, Pergamon Press, New York, 1964.

[26] M.Yu. Barabanenkov, Yu.N. Barabanenkov, S.A. Nikitov, Near field introscopy of two dimensional nonhomogeneous left-handed material slab, Proc. SPIE 8083: 808305, 2011

[27] D. Schuring, J.J. Mock, B.J. Justice, S.A. Cummer, J.B. Pendry, A.F. Starr, D.R. Smith, Metamaterial electromagnetic cloak at microwave frequencies, Science 314: 977-980, 2006.

[28] B. Zhang, Bae-Ian Wu, Electromagnetic detection of a perfect invisibility cloak, Phys. Rev. Lett. 103: 243901, 2009 